\title{
OPEN Potentially preventable hospital readmissions after patients' first stroke in Taiwan
}

\author{
Ling-Jan Chiou ${ }^{1} \&$ Hui-Chu Lang ${ }^{2 \bowtie}$
}

Readmission is an important indicator of the quality of care. The purpose of this study was to explore the probabilities and predictors of 30-day and 1-year potentially preventable hospital readmission (PPR) after a patient's first stroke. We used claims data from the National Health Insurance (NHI) from 2010 to 2018. Multinomial logistic regression was used to assess the predictors of 30-day and 1-year PPR. A total of 41,921 discharged stroke patients was identified. We found that hospital readmission rates were $15.48 \%$ within 30-days and $47.25 \%$ within 1-year. The PPR and non-PPR were $9.84 \%(4123)$ and $5.65 \%$ (2367) within 30-days, and $30.65 \%(12,849)$ and $16.60 \%$ (6959) within 1-year, respectively. The factors of older patients, type of stroke, shorter length of stay, higher Charlson Comorbidity Index (CCI), higher stroke severity index (SSI), regional hospital, public and private hospital, and hospital in the lower urbanized area were associated significantly with the 30-day PPR. In addition, the factors of male, hospitalization year, and monthly income were associated significantly with 1-year PPR. The ORs of long-term PPR showed a decreasing trend since implementing the national health insurance post-acute care (PAC) program in 2014 and a dramatic drop in 2018 after the government expanded the long-term care plan-LTC 2.0 in 2017 . The results showed that better discharge planning, implementing post-acute care programs and long-term care plan-LTC 2.0 may benefit the care of stroke patients and help reduce long-term readmission in Taiwan.

As an important indicator of the quality of care, readmissions may occur because of events or conditions in the initial hospital stay, such as poor clinical care and poor coordination of services during hospitalization ${ }^{1,2}$, incomplete treatment of the underlying problem, and/or the development of a complication that becomes evident only after discharge $e^{3}$. Stroke patients have a high probability of being readmitted to the hospital after discharge. In most studies, 30-days post-discharge was identified as the most common readmission period ${ }^{4}$. The extant literature has provided the frequency of hospital readmission, ranging from 6.5 to $24.3 \%$ within 30 -days ${ }^{4-8}$, and from 31 to $49 \%$ within 1 -year ${ }^{9-11}$.

However, studies have demonstrated that some of these readmissions are unavoidable, even with optimal care $^{12}$. Hospital readmission has also been highlighted as a source of significant healthcare expenditure and may constitute a potential target for cost savings, which supports the logic of using all-cause readmissions as a hospital performance metric ${ }^{13}$. Indeed, if readmission rates are considered a key indicator of hospital quality of care, readmissions can be identified as potentially preventable and/or unpreventable based upon clinical evidence criteria.

A readmission is defined as a subsequent hospitalization in an acute care hospital that follows a prior acute care admission within a specific time interval. Based upon administrative data, the potentially preventable readmission (PPR) method is used commonly to identify hospital readmissions that may indicate problems with a prior admission, and therefore be potentially preventable ${ }^{14}$. PPRs are those readmissions that could be avoided potentially given better clinical management, better stabilization of patients' prior discharge, appropriate discharge planning, better outpatient treatment post-discharge, and resources at home that are sufficient to meet patients' needs ${ }^{15}$. Overall, PPRs are events that could have been prevented with a better quality of hospital care, community care, and/or home care.

PPRs were defined according to AHRQ Prevention Quality Indicators (PQIs). The U.S. Agency for Healthcare Research and Quality (AHRQ) has used PQIs to define PPRs ${ }^{16}$. These PQIs constitute a set of evidence-based measures that use hospital inpatient administrative data to identify avoidable hospitalizations ${ }^{8}$. They have been employed widely to assess the quality of care and common ambulatory care-sensitive conditions ${ }^{15,17-21}$, many of which are related to readmission risk factors after stroke ${ }^{9,15}$.

${ }^{1}$ Department of Health Business Administration, Department of Nursing, and Department of Oral Hygiene, Meiho University, Pingtung County, Taiwan. ${ }^{2}$ Institute of Hospital and Health Care Administration, National Yang Ming Chiao Tung University, 155, Sec.2, Li-Nong St., Taipei 112, Taiwan. ${ }^{\circledR}$ email: hclang@nycu.edu.tw 


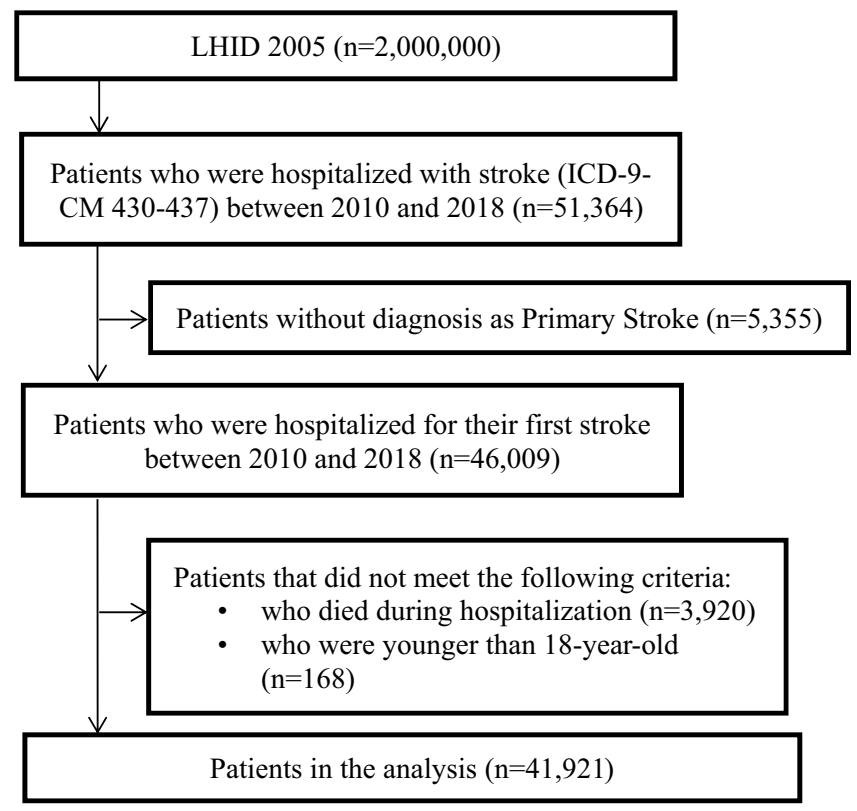

Figure 1. Flow chart of the data processing.

Patient characteristics, social circumstances, health systems, clinical care processes, and health outcomes are potential factors in readmission after a stroke ${ }^{5}$. Predictors may vary and reflect different underlying mechanisms of the causes of readmission. Bjerkreim et al. ${ }^{11}$ found that the most frequent causes of readmission were infections, recurrent ischemic stroke, other cardiovascular events, and events associated with primary stroke. Patients readmitted early had a shorter length of index admission, poorer physical function, higher frequencies of an atherosclerotic etiology of index stroke, atrial fibrillation, and complications attributable to infection during index admission compared to patients readmitted late. Late readmission was correlated with older age and prior myocardial infarction. Readmissions within a short time post-discharge may indicate poor clinical care, unresolved problems at initial discharge, the quality of immediate post-hospital care, and a more chronically ill population ${ }^{22}$.

The strategies used to prevent short-term and long-term PPR should differ. It is important for organizations to identify the managerial strategies necessary to reduce patient readmission after stroke to improve the quality of care and save costs ${ }^{4,23,24}$. Nevertheless, few studies have targeted PPR after stroke using nationally-based data and few have compared the short- (30-day) and long-term (1-year) PPR after a patient's first stroke. Thus, this study was designed to explore the preventable and non-preventable predictive factors that may influence readmission using nationwide population-based data.

\section{Materials and methods}

Data source. This study employed a population-based retrospective cohort study design. We used claims data derived from the National Health Insurance (NHI), Taiwan (LHID2005: Longitudinal Health Insurance Database 2005). This database consists of a national representative sample of one million individuals from the population of Taiwan overall. There were no statistically significant differences when age, sex, or insurance premium distributions of all NHI-enrolled individuals were compared ${ }^{25}$. The Taipei Veterans General Hospital Institutional Review Board reviewed and approved the study proposal (VGHIRB No. 2015-05-006BC\#4). The database contained no identifiable personal information; hence waiver of informed content was granted. We confirm that all experiments were performed in accordance with relevant guidelines and regulations.

Sample selection. We included patients hospitalized for their first-ever stroke (ICD-9-CM 430-437) between 2010 and 2018 who were examined within 30 days with computed tomography (CT) or magnetic resonance imaging (MRI). We excluded patients with a stroke diagnosis before the index date, those who died during hospitalization, discharged themselves voluntarily, were transferred, had fewer than three outpatient visits within one year after discharge, had no insurance record, and were younger than 18-years-old. The final sample comprised 41,921 patients. The sample selection procedure is shown in Fig. 1.

Causes of readmission. Preventable readmissions were defined according to the Agency for Healthcare Research and Quality (AHRQ) Prevention Quality Indicators (PQIs) ${ }^{8,16}$, which include chronic lung condition indicators (chronic obstructive pulmonary disease and adult asthma), diabetes-related preventable conditions (short- and long-term complications, and uncontrolled diabetes), cardiovascular-related indicators (hypertension, congestive heart failure, and angina without procedure), and acute condition indicators (dehydration, bacterial pneumonia, and urinary tract infection) (Supplementary Table S1). Non-PPR was defined as readmission 
after initial admission with a stroke diagnosis where the aforementioned diseases were not diagnosed at the time of readmission. Patients who were not readmitted to the hospital were defined as having no readmission.

Covariates and subgroups. The covariates in this study included sex, age group, year of first admission for stroke, length of stay (LOS), stroke type (ISC: ischaemic stroke (codes 433-434), ICH: intracerebral hemorrhage (codes 431-432), SAH: subarachnoid hemorrhage (codes 430), and other (codes 435-437), monthly income based upon the NHI premium each patient paid, which was used as a proxy for income, hospital level, ownership, and region, and urbanization level, in which level 1 represents the most urbanized area and level 5 the least ${ }^{26}$. A modified version of the Charlson Comorbidity Index (CCI) was used to summarize comorbidities ${ }^{27,28}$. In the 1-year follow-up, the LOS included the initial hospitalization and hospital stays later during the year.

The comorbidities with PPR and non-PPR after discharge post-stroke were calculated according to 29 diagnosed diseases (Supplementary Table S2), which include any primary and secondary diagnosed conditions in outpatient or inpatient data during the period between the first admission for stroke and readmission post-discharge. The items included in the Stroke Severity Index (SSI) essentially reflect the management of stroke-related complications, and are generally correlated with stroke severity and other accompanying neurological deficits. We extracted the above claims information from the inpatient claims database at first admission for stroke and then computed each patient's SSI. Following a previous study, patients were categorized as having mild (SSI $\leq 5)$, moderate (SSI 5 to $\leq 12)$, or severe $($ SSI $>12)$ stroke $^{29}$.

Data analysis. Descriptive statistics were used to summarize all of the covariates considered in this study, in which categorical variables were analyzed using Pearson's Chi-squared test, and continuous variables (LOS) were analyzed using the $t$-test. In this study, separate models were built to examine the covariates associated with readmission status within 30-days and 1-year. Multinomial logistic regression (MLR) was performed to determine the association between related factors and readmissions. Three levels were defined for the dependent variable, readmission status: PPR patients, non-PPR patients, and no-readmission patients. Among them, no readmission was set as the reference level. The MLR results were presented as odds ratios (ORs), $95 \%$ confidence intervals (95\% CIs), and $p$-values. Statistical significance was set at $p<0.05$. All statistical analyses were performed with SAS software v. 9.4 (SAS Institute Inc., Cary, NC, U.S.A.).

Ethics approval. Institutional Ethical Review (VGHIRB No. 2015-05-006BC\#4).

Consent for publication. All the authors have agreed this publication.

\section{Results}

41,921 discharged stroke patients in total were identified during the study period. Table 1 shows the summary statistics of all covariates for the PPR, non-PPR, and non-readmission groups. Of these patients, 6490 (15.48\%) were readmitted within 30-days, and 19,808 (47.25\%) were readmitted within 1-year. Among them, the readmission rates for PPR and non-PPR were 4123 (9.84\%) and 2367 (5.65\%) within 30-days, and 12,849 (30.65\%) and $6959(16.60 \%)$ within 1-year, respectively.

The comparison results showed the significant covariates associated with readmission status within 30-days: age, monthly income, year, stroke type, LOS, CCI, SSI, hospital level, hospital ownership, and urbanization. For both 30-day and 1-year readmissions, the PPR rate of these significant factors was more likely to be higher than non-PPR, and the highest PPR rate of subgroups among the significant factors were one patient $80+$, patients with a monthly income of NT $\$ 19,048-21,900$, those treated in 2010 and 2012, those with ICH, those with CCI $7+$, those with severe SSI, and those treated at district, private, and the least urbanized area hospitals.

The mean LOS within 30-days was $11.51(\mathrm{SD}=9.59)$ for PPR and $11.25(\mathrm{SD}=9.75)$ for non-PPR and within 1 -year were $18.56(S D=22.97)$ for PPR and $19.24(S D=25.08)$ for non-PPR. The standard deviation of LOS within 1-year appeared to be large, which indicates that the LOS values were distributed over a broader range.

The results of the multinomial logistic regression are shown in Table 2. Compared to no readmission, patients 45-64, 65-69, 70-79, and 80+ years of age vs. those 18-44 years had ORs of 1.29, 1.33, 1.43, and 1.61 for 30-day PPR, and 1.20, 1.39, 1.66, and 2.37 for 1-year PPR. In addition, these ORs indicated that older patients have a higher probability of PPR. Compared to no readmission, within 30-days, LOS had ORs of 0.97 (95\% CI [0.97-0.97]) and 0.97 (95\% CI [0.97-0.97]) for PPR and non-PPR readmission, respectively. Within 1-year, LOS had an OR of 1.01 (95\% CI [1.01-1.01]) and 1.01 (95\% CI [1.01-1.01]) for PPR and non-PPR readmission, respectively. These ORs indicated that a shorter length of stay was associated with readmission within 30-days and had a greater effect on readmission within 1-year. Further, age played a key role in readmission.

Within 30-days, the CCI level of 7+ and 4-6 (OR 1.75, 95\% CI [1.48-2.06] and OR 1.25, 95\% CI [1.12-1.39]), SSI levels of moderate and severe (OR 1.84, 95\% CI [1.63-2.09] and OR 3.69, 95\% CI [3.34-4.07]), treatment at a regional hospital (OR 1.37, 95\% CI [1.21-1.54]) and hospital ownership, public and private (OR 1.11, 95\% CI [1.02-1.21] and OR 1.12, 95\% CI [1.01-1.23]), all types of stroke, all age levels and urbanization, were associated significantly with readmission. Similarly, all CCI levels (OR 1.40, 95\% CI [1.33-1.48]; OR 2.25, 95\% CI [2.09-2.42] and OR 3.81, 95\% CI [3.33-4.36]), moderate and severe SSI levels (OR 1.60, 95\% CI [1.46-1.75] and OR 3.27, 95\% CI [3.03-3.53]), treatment at a medical center or regional hospital (OR 1.08, 95\% CI [1.02-1.14] and OR 1.40, 95\% CI [1.29-1.53]), and type of stroke, all age levels, and urbanization had a significant effect on readmission within 1-year. In addition to these covariates, male, hospitalization year, monthly income, and treatment at a hospital in the central area of Taiwan, also affected readmission significantly. Moreover, direct trends were discernable for age, CCI, SSI, and urbanization for PPR within 1-year, and age and SSI for PPR 


\begin{tabular}{|c|c|c|c|c|c|c|c|c|c|c|c|c|c|c|}
\hline \multirow[b]{3}{*}{ Variable } & \multicolumn{7}{|c|}{30 days } & \multicolumn{7}{|l|}{1 year } \\
\hline & \multicolumn{2}{|l|}{ PPR } & \multicolumn{2}{|c|}{ Non-PPR } & \multicolumn{2}{|c|}{ Non-readmission } & \multirow{2}{*}{\begin{tabular}{|l|} 
Chi-squared \\
$p$-value \\
\end{tabular}} & \multicolumn{2}{|l|}{ PPR } & \multicolumn{2}{|c|}{ Non-PPR } & \multicolumn{2}{|c|}{ Non-readmission } & \multirow{2}{*}{\begin{tabular}{|l|} 
Chi-squared \\
$p$-value \\
\end{tabular}} \\
\hline & n & $\%$ & $\mathbf{n}$ & $\%$ & $\mathbf{n}$ & $\%$ & & $\mathbf{n}$ & $\%$ & $\mathbf{n}$ & $\%$ & $\mathbf{n}$ & $\%$ & \\
\hline $\mathrm{N}$ & 4123 & 9.8 & 2367 & 5.7 & 35,431 & 84.5 & & 12,849 & 30.7 & 6959 & 16.6 & 22,113 & 52.8 & \\
\hline \multicolumn{15}{|l|}{ Sex } \\
\hline Male & 2444 & 9.9 & 1447 & 5.9 & 20,790 & 84.2 & 0.054 & 7440 & 30.1 & 4223 & 17.1 & 13,018 & 52.8 & $<0.001$ \\
\hline Female & 1679 & 9.7 & 920 & 5.3 & $\mid 14,641$ & \begin{tabular}{|l|}
84.9 \\
\end{tabular} & & 5409 & 31.4 & 2736 & $\mid 15.9$ & 9095 & \begin{tabular}{|l|}
52.8 \\
\end{tabular} & \\
\hline \multicolumn{15}{|l|}{ Age } \\
\hline $18-44$ & 184 & 7.3 & 225 & 8.9 & 2121 & 83.8 & $<0.001$ & 499 & 19.7 & 595 & 23.5 & 1436 & 56.8 & $<0.001$ \\
\hline $45-64$ & 1239 & 8.9 & 788 & 5.7 & 11,873 & 85.4 & & 3449 & 24.8 & 2211 & 15.9 & 8240 & 59.3 & \\
\hline $65-69$ & 426 & 9.1 & 259 & 5.5 & 3994 & \begin{tabular}{|l|}
85.4 \\
\end{tabular} & & 1314 & 28.1 & 750 & 16.0 & 2615 & 55.9 & \\
\hline 70-79 & 1071 & 10.1 & 550 & 5.2 & 9013 & 84.8 & & 3469 & 32.6 & 1690 & 15.9 & 5475 & 51.5 & \\
\hline$\geqq 80$ & 1203 & 11.8 & 545 & 5.4 & 8430 & \begin{tabular}{|l|}
82.8 \\
\end{tabular} & & 4118 & 40.5 & 1713 & \begin{tabular}{|l|}
16.8 \\
\end{tabular} & 4347 & 42.7 & \\
\hline \multicolumn{15}{|l|}{ Monthly income } \\
\hline$\leqq 19,047$ & 1333 & 10.0 & 768 & 5.8 & 11,190 & 84.2 & $<0.001$ & 4219 & 31.7 & 2257 & 17.0 & 6815 & 51.3 & $<0.001$ \\
\hline $19,048-21,900$ & 1659 & 10.5 & 862 & 5.5 & 13,243 & 84.0 & & 5111 & 32.4 & 2628 & 16.7 & 8025 & 50.9 & \\
\hline$>21,900$ & 1131 & 8.8 & 737 & 5.7 & \begin{tabular}{|l|}
10,998 \\
\end{tabular} & \begin{tabular}{|l|}
85.5 \\
\end{tabular} & & 3519 & 27.4 & 2074 & 16.1 & 7273 & 56.5 & \\
\hline Year & & & & & & & & & & & & & & \\
\hline 2010 & 516 & 10.5 & 222 & 4.5 & 4196 & 85.0 & $<0.001$ & 1671 & 33.9 & 714 & 14.5 & 2549 & 51.7 & $<0.001$ \\
\hline 2011 & 463 & 9.1 & 235 & 4.6 & 4397 & 86.3 & & 1669 & 32.8 & 765 & 15.0 & 2661 & 52.2 & \\
\hline 2012 & 503 & \begin{tabular}{|l|}
10.4 \\
\end{tabular} & 235 & 4.9 & 4106 & \begin{tabular}{|l|}
84.8 \\
\end{tabular} & & 1665 & 34.4 & 702 & 14.5 & 2477 & 51.1 & \\
\hline 2013 & 466 & 9.6 & 234 & 4.8 & 4132 & 85.5 & & 1580 & 32.7 & 762 & 15.8 & 2490 & 51.5 & \\
\hline 2014 & 466 & 9.9 & 255 & 5.4 & 3981 & \begin{tabular}{|l|}
84.7 \\
\end{tabular} & & 1491 & 31.7 & 769 & 16.4 & 2442 & 51.9 & \\
\hline 2015 & 452 & 9.6 & 269 & 5.7 & 4001 & \begin{tabular}{|l|}
84.7 \\
\end{tabular} & & 1406 & 29.8 & 782 & 16.6 & 2534 & 53.7 & \\
\hline 2016 & 426 & 9.9 & 289 & 6.7 & 3597 & 83.4 & & 1261 & 29.2 & 888 & 20.6 & 2163 & 50.2 & \\
\hline 2017 & 430 & \begin{tabular}{|l|}
10.1 \\
\end{tabular} & 321 & 7.6 & 3501 & \begin{tabular}{|l|}
82.3 \\
\end{tabular} & & 1200 & 28.2 & 917 & 21.6 & 2135 & 50.2 & \\
\hline 2018 & 401 & 9.5 & 307 & 7.3 & 3520 & \begin{tabular}{|l|}
83.3 \\
\end{tabular} & & 906 & 21.4 & 660 & 15.6 & 2662 & 63.0 & \\
\hline Stroke type & & & & & & & & & & & & & & \\
\hline ISC & 2690 & 9.7 & 1476 & 5.3 & 23,635 & 85.0 & $<0.001$ & 8560 & 30.8 & 4378 & 15.8 & 14,863 & 53.5 & $<0.001$ \\
\hline $\mathrm{ICH}$ & 885 & 13.1 & 497 & 7.4 & 5384 & 79.6 & & 2348 & 34.7 & 1367 & 20.2 & 3051 & 45.1 & \\
\hline Other & 477 & 7.2 & 332 & 5.0 & 5821 & \begin{tabular}{|l|}
87.8 \\
\end{tabular} & & 1733 & 26.1 & 1053 & 15.9 & 3844 & 58.0 & \\
\hline SAH & 71 & 9.8 & 62 & 8.6 & 591 & 81.6 & & 208 & 28.7 & 161 & 22.2 & 355 & 49.0 & \\
\hline LOS (mean, SD) & 11.51 & 9.6 & 11.25 & 9.8 & 16.3 & 35.8 & $<0.001$ & 18.56 & 23.0 & 19.24 & 25.1 & 12.63 & 39.6 & $<0.001$ \\
\hline $\mathrm{CCI}$ & & & & & & & & & & & & & & \\
\hline 0 & 1185 & 9.2 & 736 & 5.7 & 10,943 & 85.1 & $<0.001$ & 3216 & 25.0 & 1951 & 15.2 & 7697 & 59.8 & $<0.001$ \\
\hline $1-3$ & 2065 & 9.6 & 1119 & 5.2 & 18,390 & 85.2 & & 6673 & 30.9 & 3353 & 15.5 & 11,548 & 53.5 & \\
\hline $4-6$ & 660 & 11.1 & 349 & 5.9 & 4949 & 83.1 & & 2311 & 38.8 & 1191 & 20.0 & 2456 & 41.2 & \\
\hline$\geqq 7$ & 213 & 14.0 & 163 & 10.7 & 1149 & 75.3 & & 649 & 42.6 & 464 & 30.4 & 412 & 27.0 & \\
\hline SSI & & & & & & & & & & & & & & \\
\hline Mild & 2855 & 8.4 & 1653 & 4.9 & 29,402 & 86.7 & $<0.001$ & 9304 & 27.4 & 5132 & 15.1 & 19,474 & 57.4 & $<0.001$ \\
\hline Moderate & 364 & 13.4 & 198 & 7.3 & 2150 & 79.3 & & 1002 & 37.0 & 503 & 18.6 & 1207 & 44.5 & \\
\hline Severe & 904 & 17.1 & 516 & 9.7 & 3879 & 73.2 & & 2543 & 48.0 & 1324 & 25.0 & 1432 & 27.0 & \\
\hline Hospital level & & & & & & & & & & & & & & \\
\hline Medical center & 1368 & 9.1 & 863 & 5.8 & 12,757 & 85.1 & $<0.001$ & 4279 & 28.6 & 2672 & \begin{tabular}{|l|}
17.8 \\
\end{tabular} & 8037 & 53.6 & $<0.001$ \\
\hline Regional hospital & 1965 & 9.3 & 1102 & 5.2 & $\mid 18,014$ & 85.5 & & 6464 & 30.7 & 3302 & 15.7 & 11,315 & \begin{tabular}{|l|}
53.7 \\
\end{tabular} & \\
\hline District hospital & 789 & 13.5 & 402 & 6.9 & 4646 & 79.6 & & 2103 & 36.0 & 983 & 16.8 & 2751 & 47.1 & \\
\hline Hospital ownershi & & & & & & & & & & & & & & \\
\hline Public & 1217 & 10.2 & 682 & 5.7 & 9984 & 84.0 & $<0.001$ & 3696 & 31.1 & 2019 & 17.0 & 6168 & 51.9 & 0.072 \\
\hline Private & 898 & 10.9 & 493 & 6.0 & 6878 & 83.2 & & 2589 & 31.3 & 1343 & 16.2 & 4337 & 52.5 & \\
\hline Legal person & 2007 & 9.2 & 1192 & 5.5 & 18,557 & 85.3 & & 6561 & 30.2 & 3595 & 16.5 & 11,600 & 53.3 & \\
\hline Urbanization leve & & & & & & & & & & & & & & \\
\hline 1 & 543 & 7.8 & 330 & 4.8 & 6075 & 87.4 & $<0.001$ & 1972 & 28.4 & 1114 & 16.0 & 3862 & 55.6 & $<0.001$ \\
\hline 2 & 1211 & 10.5 & 746 & 6.5 & 9608 & 83.1 & & 3437 & 29.7 & 1990 & 17.2 & 6138 & 53.1 & \\
\hline 3 & 879 & 9.1 & 495 & 5.1 & 8290 & \begin{tabular}{|l|}
85.8 \\
\end{tabular} & & 2931 & 30.3 & 1571 & 16.3 & 5162 & 53.4 & \\
\hline 4 & 705 & 10.0 & 398 & 5.7 & 5925 & 84.3 & & 2234 & 31.8 & 1173 & 16.7 & 3621 & 51.5 & \\
\hline 5 & 776 & 11.7 & 391 & 5.9 & 5453 & 82.4 & & 2257 & 34.1 & 1093 & 16.5 & 3270 & \begin{tabular}{|l|}
49.4 \\
\end{tabular} & \\
\hline
\end{tabular}

Table 1. Factors associated with potentially preventable readmission (PPR), non- preventable readmission (non-PPR) and non-readmission patients within 30 days/1 year all after stroke $(n=41,921)$. 


\begin{tabular}{|c|c|c|c|c|c|c|c|c|}
\hline \multirow[b]{3}{*}{ Table } & \multicolumn{4}{|l|}{30 days } & \multicolumn{4}{|l|}{1 year } \\
\hline & \multicolumn{2}{|c|}{$\begin{array}{l}\text { PPR vs. without } \\
\text { readmission }\end{array}$} & \multicolumn{2}{|c|}{$\begin{array}{l}\text { Non-PPR vs. without } \\
\text { readmission }\end{array}$} & \multicolumn{2}{|c|}{$\begin{array}{l}\text { PPR vs. without } \\
\text { readmission }\end{array}$} & \multicolumn{2}{|c|}{$\begin{array}{l}\text { Non-PPR vs. without } \\
\text { readmission }\end{array}$} \\
\hline & \begin{tabular}{|l|} 
OR \\
\end{tabular} & 95\% CI & OR & $95 \% \mathrm{CI}$ & OR & 95\% CI & OR & 95\% CI \\
\hline \multicolumn{9}{|l|}{ Sex } \\
\hline \multicolumn{9}{|l|}{ Female } \\
\hline Male & 1.05 & $(0.98-1.12)$ & 1.08 & $(0.99-1.18)$ & ${ }^{* *} 1.07$ & $(1.02-1.13)$ & $* * * 1.14$ & $(1.08-1.21)$ \\
\hline \multicolumn{9}{|l|}{ Age } \\
\hline \multicolumn{9}{|l|}{$18-44$} \\
\hline $45-64$ & $* * 1.29$ & $(1.09-1.52)$ & ${ }^{* * *} 0.68$ & $(0.58-0.80)$ & ${ }^{* *} 1.20$ & $(1.07-1.34)$ & ${ }^{* * *} 0.66$ & $(0.59-0.74)$ \\
\hline $65-69$ & $* * 1.33$ & $(1.10-1.60)$ & ${ }^{* * *} 0.66$ & $(0.54-0.88)$ & $* * * 1.39$ & $(1.22-1.58)$ & $* * * 0.67$ & $(0.59-0.77)$ \\
\hline $70-79$ & ${ }^{* * *} 1.43$ & $(1.20-1.69)$ & ${ }^{* * *} 0.62$ & $(0.52-0.74)$ & ${ }^{* * *} 1.66$ & $(1.48-1.86)$ & $* * * 0.71$ & $(0.64-0.80)$ \\
\hline$\geqq 80$ & ${ }^{* * *} 1.61$ & $(1.35-1.91)$ & ${ }^{* * *} 0.62$ & $(0.52-0.74)$ & $* * * 2.37$ & $(2.11-2.67)$ & ${ }^{*} 0.88$ & $(0.78-0.99)$ \\
\hline \multicolumn{9}{|l|}{ Monthly income } \\
\hline \multicolumn{9}{|l|}{$19,048-21,900$} \\
\hline$\leqq 19,047$ & 1.07 & $(1.00-1.18)$ & 1.02 & $(0.92-1.14)$ & $* * * 1.11$ & $(1.04-1.17)$ & ${ }^{*} 1.08$ & $(1.01-1.16)$ \\
\hline$>21,900$ & 1.07 & $(0.99-1.17)$ & 0.99 & $(0.89-1.11)$ & ${ }^{*} 1.07$ & $(1.01-1.13)$ & ${ }^{*} 1.09$ & $(1.02-1.18)$ \\
\hline \multicolumn{9}{|l|}{ Year } \\
\hline \multicolumn{9}{|l|}{2010} \\
\hline 2011 & ${ }^{*} 0.84$ & $(0.74-0.97)$ & 1 & $(0.83-1.21)$ & ${ }^{*} 0.89$ & $(0.81-0.98)$ & 0.96 & $(0.85-1.08)$ \\
\hline 2012 & 0.99 & $(0.86-1.13)$ & 1.07 & $(0.88-1.30)$ & 0.97 & $(0.88-1.06)$ & 0.95 & $(0.84-1.07)$ \\
\hline 2013 & 0.9 & $(0.78-1.03)$ & 1.05 & $(0.86-1.27)$ & 0.92 & $(0.84-1.00)$ & 1.04 & $(0.92-1.17)$ \\
\hline 2014 & 0.95 & $(0.83-1.09)$ & 1.19 & $(0.99-1.44)$ & ${ }^{*} 0.89$ & $(0.81-0.98)$ & 1.06 & $(0.94-1.19)$ \\
\hline 2015 & 0.95 & $(0.83-1.09)$ & ${ }^{* *} 1.31$ & $(1.09-1.57)$ & $* * * 0.81$ & $(0.74-0.90)$ & 1.06 & $(0.94-1.19)$ \\
\hline 2016 & 1.04 & $(0.90-1.19)$ & $* * * 1.66$ & $(1.38-2.00)$ & ${ }^{* *} 0.87$ & $(0.79-0.96)$ & $* * * 1.44$ & $(1.28-1.62)$ \\
\hline 2017 & 1.07 & $(0.93-1.23)$ & ${ }^{* * *} 1.88$ & $(1.57-2.26)$ & $* * * 0.84$ & $(0.76-0.93)$ & $* * * 1.52$ & $(1.35-1.70)$ \\
\hline 2018 & $* * * 0.99$ & $(0.85-1.14)$ & $* * * 1.80$ & $(1.50-2.16)$ & $* * * 0.49$ & $(0.45-0.55)$ & $* * * 0.85$ & $(0.75-0.96)$ \\
\hline \multicolumn{9}{|l|}{ Stroke type } \\
\hline \multicolumn{9}{|l|}{ Other type } \\
\hline ISC & $* * * 1.44$ & $(1.30-1.60)$ & 1.06 & $(0.94-1.21)$ & $* * * 1.20$ & $(1.12-1.28)$ & 0.93 & $(0.86-1.01)$ \\
\hline $\mathrm{ICH}$ & $* * * 1.97$ & $(1.73-2.25)$ & ${ }^{* *} 1.29$ & $(1.10-1.52)$ & $* * * 1.40$ & $(1.28-1.53)$ & 1.08 & $(0.97-1.20)$ \\
\hline SAH & ${ }^{* *} 1.48$ & (1.13-1.95) & ${ }^{*} 1.39$ & $(1.03-1.87)$ & 1.17 & $(0.97-1.43)$ & 1.17 & $(0.97-1.43)$ \\
\hline LOS & $* * * 0.97$ & $(0.97-0.97)$ & ${ }^{* * *} 0.97$ & $(0.97-0.97)$ & ${ }^{* * *} 1.01$ & $(1.01-1.01)$ & $* * * 1.01$ & $(1.01-1.01)$ \\
\hline CCI & & & & & & & & \\
\hline 0 & & & & & & & & \\
\hline $1-3$ & 1.06 & $(0.98-1.15)$ & 1.01 & $(0.91-1.12)$ & $* * * 1.40$ & $(1.33-1.48)$ & $* * * 1.28$ & $(1.19-1.36)$ \\
\hline $4-6$ & $* * * 1.25$ & $(1.12-1.39)$ & ${ }^{\star} 1.18$ & $(1.03-1.36)$ & $* * * 2.25$ & $(2.09-2.42)$ & $* * * 2.15$ & $(1.97-2.36)$ \\
\hline$\geqq 7$ & ${ }^{* * *} 1.75$ & $(1.48-2.06)$ & $* * * 2.41$ & $(1.99-2.90)$ & $* * * 3.81$ & $(3.33-4.36)$ & $* * * 4.94$ & $(4.27-5.71)$ \\
\hline SSI & & & & & & & & \\
\hline Mild & & & & & & & & \\
\hline Moderate & $* * * 1.84$ & $(1.63-2.09)$ & ${ }^{* * *} 1.98$ & $(1.68-2.32)$ & ${ }^{* * *} 1.60$ & $(1.46-1.75)$ & $* * * 1.55$ & $(1.38-1.73)$ \\
\hline Severe & $* * * 3.69$ & (3.34-4.07) & ${ }^{* * *} 4.18$ & (3.69-4.74) & $* * * 3.27$ & $(3.03-3.53)$ & $* * * 3.18$ & $(2.90-3.47)$ \\
\hline Hospital level & & & & & & & & \\
\hline District hospital & & & & & & & & \\
\hline Medical center & 0.96 & $(0.88-1.04)$ & ${ }^{*} 0.90$ & $(0.81-1.00)$ & ${ }^{* *} 1.08$ & $(1.02-1.14)$ & ${ }^{* *} 0.92$ & $(0.86-0.98)$ \\
\hline Regional hospital & $* * * 1.37$ & $(1.21-1.54)$ & ${ }^{*} 1.22$ & $(1.04-1.42)$ & $* * * 1.40$ & $(1.29-1.53)$ & ${ }^{\star} 1.12$ & $(1.00-1.24)$ \\
\hline Hospital ownersh & & & & & & & & \\
\hline Legal person & & & & & & & & \\
\hline Public & ${ }^{*} 1.11$ & $(1.02-1.21)$ & 1.02 & $(0.91-1.13)$ & 1.03 & $(0.97-1.10)$ & 1.04 & $(0.96-1.11)$ \\
\hline Private & ${ }^{*} 1.12$ & $(1.01-1.23)$ & 0.99 & $(0.87-1.13)$ & 1.03 & $(0.96-1.11)$ & 0.98 & $(0.90-1.07)$ \\
\hline Urbanization leve & & & & & & & & \\
\hline 1 & & & & & & & & \\
\hline 2 & ${ }^{* * *} 1.38$ & $(1.24-1.55)$ & ${ }^{* * *} 1.33$ & $(1.15-1.53)$ & ${ }^{* *} 1.13$ & $(1.05-1.21)$ & ${ }^{*} 1.10$ & $(1.01-1.21)$ \\
\hline 3 & ${ }^{*} 1.17$ & $(1.03-1.33)$ & 0.98 & $(0.83-1.16)$ & ${ }^{\star} 1.13$ & $(1.04-1.24)$ & 1.06 & $(0.95-1.18)$ \\
\hline 4 & $* * * 1.28$ & $(1.12-1.46)$ & 1.18 & $(0.99-1.40)$ & ${ }^{* *} 1.15$ & $(1.05-1.26)$ & $* * * 1.15$ & $(1.03-1.28)$ \\
\hline 5 & $* * * 1.34$ & $(1.16-1.55)$ & 1.34 & $(1.16-1.55)$ & ${ }^{* *} 1.24$ & $(1.12-1.37)$ & 1.21 & $(1.07-1.36)$ \\
\hline
\end{tabular}

Table 2. Factors associated with PPR and non-PPR $v s$. non-readmission after stroke (multinomial logistic regression). ${ }^{*} p<0.05,{ }^{* *} p<0.01,{ }^{* *} p<0.001$. 
within 30-days. Further, Fig. 2 shows the forest plot of the odds ratios and 95\% confidence intervals for factors associated with 30-day and 1-year PPR.

\section{Discussion}

Studies have found that the rates of hospital readmissions after a stroke ranged from 6.5 to $24.3 \%$ within 30 -days ${ }^{5}$ and 31 to $49 \%$ within 1 -year ${ }^{11}$. However, not all readmissions are considered "potentially preventable" ${ }^{\text {" }}$. A review paper reported that preventable readmissions ranged from 14 to $23 \%$ within 30 -days and from 48 to $59 \%$ within 1-year based upon older patients or general medical patients ${ }^{15,31}$. Another study estimated that the 1-year cumulative risks of readmission for ischemic stroke patients in Taiwan were $34.1 \%, 44.7 \%$, and $62.9 \%$ for patients with mild, moderate, or severe stroke, respectively ${ }^{32}$. In this study, we determined that hospital readmission rates were $15.48 \%$ within 30 -days and $47.25 \%$ within 1 -year; the PPRs based upon the PQI definition were $9.84 \%$ within 30 -days and $30.65 \%$ within 1-year using population-based data in Taiwan. Because our study included only patients older than 18 , the readmission rates shown should be the upper bound.

Mittal et al. found that 41 (7.6\%) of 537 acute ischemic stroke (AIS) patients were readmitted within 30-days post-stroke, and $2.8 \%$ among them were $\mathrm{PPR}^{23}$. Based upon 79 unplanned readmissions, an investigation at a Hong Kong geriatric center found that only 15 cases (19\%) were avoidable ${ }^{33}$. These variations in PPR rates may be associated with age, patient diagnosis, duration of follow-up, methodology, and factors related to the mixtures of case diagnoses ${ }^{15}$. Nakagawa et al. found in a multiethnic population in Hawaii that $840(8.4 \%)$ of 10,050 patients with any type of stroke-related hospitalization had 30-day PPR. Some studies have demonstrated that a higher readmission rate may be attributable to language barriers that affect receiving hospital care and/ or accessing post-hospital care co,34. $^{30}$.

The extant literature has reported that certain patient characteristics, such as age and socioeconomic status, were potential factors associated with readmission after stroke $e^{5,8,15}$. Our study found the same effect of age, but patients with the highest and lowest monthly income had a significantly higher rate of readmissions than those with the median income. This may indicate inequalities in healthcare and additional investigation is necessary to determine the reasons ${ }^{35}$. Regarding the age effect, the results showed that patients in the other age groups were at higher risk for short-term and long-term preventable readmissions than patients in the youngest age group. In contrast, the risk of non-preventable readmission was lower than in the youngest age group. It indicated that most readmissions in the younger age group are due to unpreventable factors.

The severity of stroke upon the first admission was also a significant predictor of 28-day readmission in Australia $^{5}$. Further, CCI was found to be associated with the 30-day PPR after stroke discharge ${ }^{30}$. In this study, we identified a direct, positive relation between age, CCI, SSI, and long-term PPR. In cases of long-term PPR, the increase in these factors was associated with increasing readmission. These findings were similar to a 234 hospital-based study in Florida, which found that PPR was related to the severity of illness and older age. In addition, their results showed that increased severity of the disease and time between admission and readmission increased readmission rates ${ }^{14}$.

After adjusting for other variables, regional hospitals showed a higher risk of PPR compared to medical centers and district hospitals. The effect of hospital-level on short- and long-term readmissions was consistent with those of previous studies ${ }^{32,36}$. We assume that medical centers provide a better quality of inpatient care ${ }^{32}$, and suggest that regional hospitals' policymakers give more attention to the quality of patient care. In addition, the fact that district hospitals had lower PPR than regional hospitals may be attributable to the implementation of the Post-Acute Care (PAC) program in Taiwan described in the next paragraph. The district hospitals received more PAC patients, which led to a decreasing readmission rate.

Our results showed that the hospitals' urbanization level was related significantly to both short- and longterm PPR; the most urbanized area had the lowest readmission rate compared to the least urbanized area. One study suggested that this may be related to the poor quality of care in rural areas ${ }^{37}$. Most discharged stroke patients still need to receive follow-up healthcare at home or in a skilled nursing or inpatient rehabilitation facility; however, those resources may not be allocated sufficiently in rural areas compared to urban areas ${ }^{38}$. As a result, the quality of post-discharge care in rural areas may be poorer than that in urban areas and have led to a higher readmission rate.

Our study demonstrated further that, compared to no-readmission patients, a 1-day increase in LOS was associated significantly with 0.97 times the risk of 30-day PPR. However, a 1-day increase in LOS was associated significantly with 1.01 times the risk of 1-year PPR. Hence, LOS may have different implications for short- and long-term PPR. This finding is consistent with that in Bjerkreim's study ${ }^{11}$. LOS' short-term effect on PPR may be explained by incomplete treatment during the index hospitalization ${ }^{14}$, and suggests the need for a better quality of care and discharge planning. On the other hand, LOS' long-term effect on PPR may be related to the severity of the stroke or comorbidities ${ }^{10,39,40}$, and suggests the need to improve the continuity of follow-up care.

The comorbidities associated with PPR diagnosed most frequently in our study were hypertension without complications, diabetes, and congestive heart failure. Previous reports have indicated that patients who were readmitted either early or later seemed to have higher frequencies of hypertension, atrial fibrillation, cerebrovascular disease, and diabetes as prior comorbidity conditions ${ }^{10,11}$. To decrease the risk of short-term PPR after discharge, our results showed that older patients, stroke type (ICH), CCI level of 4-6 and 7+, either moderate or severe SSI, and patients treated at regional, public or private, and hospitals in less urbanized areas are the groups most likely to experience a first-ever stroke, which suggest that adequate discharge planning must be provided for the first month after these patients are discharged. Although a previous study indicated that readmission reduction initiatives might not be highly effective for patients who are socioeconomically disadvantaged ${ }^{41}$, we found that more attention should be given to median-income patients to decrease readmission rates. 
A

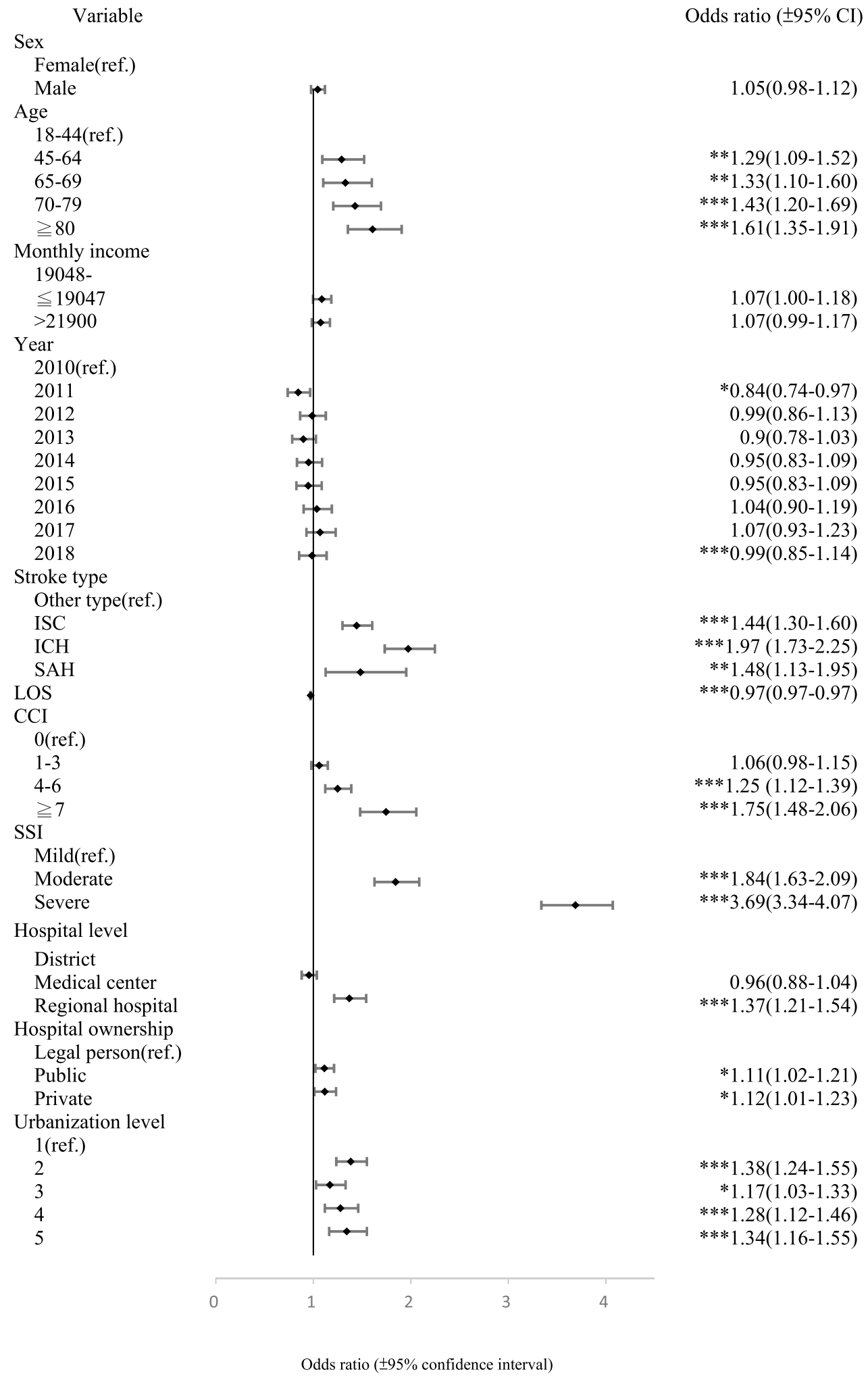

Figure 2. Forest plot displaying Odds ratios and 95\% confidence intervals for 30-day (A) and 1-year PPR (B) after multinomial logistic regression. ${ }^{*} \mathrm{p}<0.05,{ }^{* *} \mathrm{p}<0.01,{ }^{* * *} \mathrm{p}<0.001$. 


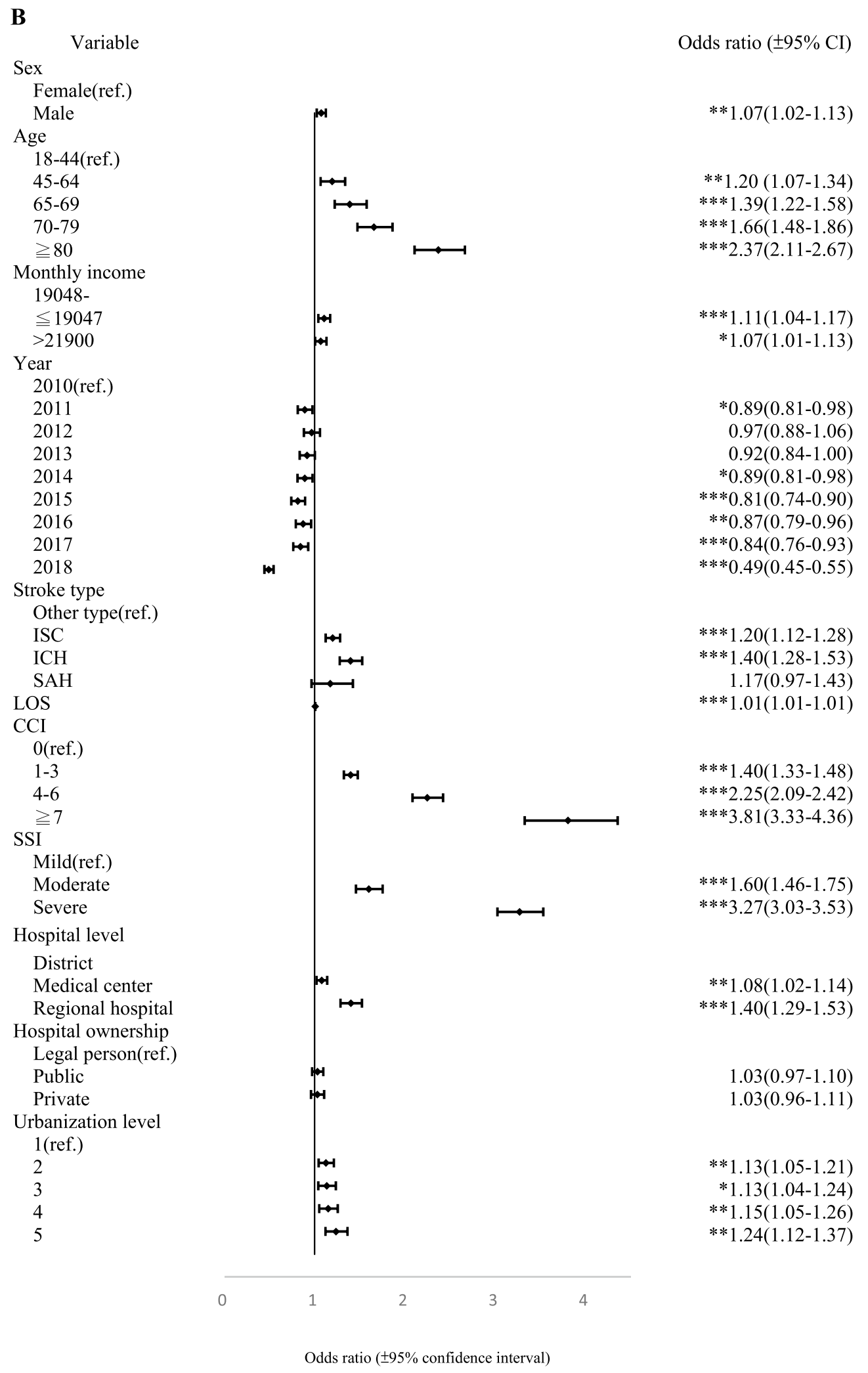

Figure 2. (continued) 
An important finding was that the ORs of long-term PPR vs. no readmission showed a decreasing trend. We believe that this is attributable to Taiwan's implementation in 2014 of the national health insurance postacute care (PAC) program for first-ever stroke patients. Patients who qualify for the PAC can receive intensive rehabilitation and integrated care within the treatment period. The PAC plan proposes to improve the incentives and review of discharge care for stroke patients in these hospitals. We suspect that the sudden drop in PPR and non-PPR readmission in 2018 might be due to the government expansion of the long-term care plan-LTC 2.0 in 2017, which encourages hospitals to provide more discharge plan services and offer more home-based medical care to the patients. The LTC-2.0 may benefit the care of stroke patients and decrease readmission. We suggest further research to verify this relationship.

PPR events may be avoided and healthcare costs reduced by improving the quality of care during the index inpatient stay and the period immediately following discharge. As a consequence, our study suggests that specific groups of patients should be targeted for PPR intervention.

\section{Limitations}

This study has certain limitations. First, it was a retrospective cohort study with data derived from Taiwan's National Health Insurance (NHI) claims database. Although we adopted rigorous definition of stroke to selection patients, overdiagnosis or underdiagnosis could not be overall excluded. In addition, data on certain important factors, such as patient behavioral characteristics, the process of care, and health-related quality of life could not be collected ${ }^{23}$. Nonetheless, compared to a hospital chart review database, the NHI claims database provides a population-based sample from a wide range of hospitals, as well as longitudinal follow-up information on readmission post-stroke. Second, the diagnosis codes' accuracy was uncertain. Therefore, the results may be limited to patients who are hospitalized with a primary discharge diagnosis of stroke in Taiwan ${ }^{36}$. Third, identifying the risk of readmission may help with interventions to reduce readmissions. However, they cannot always be considered preventable, and readmission conditions such as the risk of deterioration of the chronic pulmonary disease, diabetes complications, heart failure, new stroke events, etc., cannot be eliminated entirely. Forth, Due to the data availability, we couldn't include post-discharge death in our analysis. The results for the separate predictive model of death or potentially avoidable readmission may differ, and it is valuable for future researchers to explore it.

\section{Conclusion}

We suggest that hospital managers provide better discharge planning and post-discharge follow-up programs for these patients before and after discharge, as the combination is likely to reduce the number of PPR substantially.

\section{Data availability}

Data are sourced from Taiwan National Health Insurance (NHI). Due to legal restrictions imposed by the government of Taiwan in relation to the "Personal Information Protection Act", data cannot be made publicly available. Requests for data can be sent as a formal proposal to the NHIRD (http://nhird.nhri.org.tw).

Received: 19 October 2021; Accepted: 22 February 2022

Published online: 08 March 2022

\section{References}

1. Halfon, P. et al. Validation of the potentially avoidable hospital readmission rate as a routine indicator of the quality of hospital care. Med. Care. 44(11), 972-981 (2006).

2. Kripalani, S. et al. Deficits in communication and information transfer between hospital-based and primary care physicians: Implications for patient safety and continuity of care. JAMA 297(8), 831-841 (2007).

3. Hannan, E. L. et al. Predictors of readmission for complications of coronary artery bypass graft surgery. JAMA 290(6), 773-780 (2003).

4. Vest, J. R., Gamm, L. D., Oxford, B. A., Gonzalez, M. I. \& Slawson, K. M. Determinants of preventable readmissions in the United States: A systematic review. Implement Sci. 5(1), 88 (2010).

5. Kilkenny, M. F., Longworth, M., Pollack, M., Levi, C. \& Cadilhac, D. A. New South Wales Stroke Services Coordinating C, Agency for Clinical I: Factors associated with 28-day hospital readmission after stroke in Australia. Stroke 44(8), 2260-2268 (2013).

6. Donzé, J., Aujesky, D., Williams, D. \& Schnipper, J. L. Potentially avoidable 30-day hospital readmissions in medical patients: Derivation and validation of a prediction model. JAMA Intern. Med. 173(8), 632-638 (2013).

7. Halfon, P. et al. Measuring potentially avoidable hospital readmissions. J. Clin. Epidemiol. 55(6), 573-587 (2002).

8. Lichtman, J. H., Leifheit-Limson, E. C., Jones, S. B., Wang, Y. \& Goldstein, L. B. Preventable readmissions within 30 days of ischemic stroke among Medicare beneficiaries. Stroke 44(12), 3429-3435 (2013).

9. Bravata, D. M., Ho, S. Y., Meehan, T. P., Brass, L. M. \& Concato, J. Readmission and death after hospitalization for acute ischemic stroke: 5-year follow-up in the medicare population. Stroke 38(6), 1899-1904 (2007).

10. Lin, H. J., Chang, W. L. \& Tseng, M. C. Readmission after stroke in a hospital-based registry: Risk, etiologies, and risk factors. Neurology 76(5), 438-443 (2011).

11. Bjerkreim, A. T., Thomassen, L., Brogger, J., Waje-Andreassen, U. \& Naess, H. Causes and predictors for hospital readmission after ischemic stroke. J. Stroke Cerebrovasc. Dis. 24(9), 2095-2101 (2015).

12. van Walraven, C., Bennett, C., Jennings, A., Austin, P. C. \& Forster, A. J. Proportion of hospital readmissions deemed avoidable: A systematic review. CMAJ 183(7), E391-402 (2011).

13. Joynt, K. E. \& Jha, A. K. Thirty-day readmissions-Truth and consequences. N. Engl. J. Med. 366(15), 1366-1369 (2012).

14. Goldfield, N. I. et al. Identifying potentially preventable readmissions. Health Care Financ. Rev. 30(1), 75-91 (2008).

15. Yam, C. H. et al. Measuring and preventing potentially avoidable hospital readmissions: A review of the literature. Hong Kong Med. J. 16(5), 383-389 (2010).

16. Prevention quality indicators resources. http://www.qualityindicators.ahrq.gov/modules/pqi_resources.aspx. (accessed 2 Jan 2021).

17. Chang, C. F., Mirvis, D. M. \& Waters, T. M. The effects of race and insurance on potentially avoidable hospitalizations in Tennessee. Med. Care Res. Rev. 65(5), 596-616 (2008).

18. Davies, S. et al. Expanding the uses of AHRQ's prevention quality indicators: Validity from the clinician perspective. Med. Care. 49(8), 679-685 (2011). 
19. Kim, H., Helmer, D. A., Zhao, Z. \& Boockvar, K. Potentially preventable hospitalizations among older adults with diabetes. Am. J. Manag. Care. 17(11), e419-e426 (2011).

20. O’Neil, S. S. et al. Racial disparities in hospitalizations for ambulatory care-sensitive conditions. Am. J. Prev. Med. 38(4), 381-388 (2010).

21. Zhan, C., Miller, M. R., Wong, H. \& Meyer, G. S. The effects of HMO penetration on preventable hospitalizations. Health Serv. Res. 39(2), 345-361 (2004).

22. Lichtman, J. H. et al. Predictors of hospital readmission after stroke: A systematic review. Stroke 41(11), 2525-2533 (2010).

23. Mittal, M. K., Rabinstein, A. A., Mandrekar, J., Brown, R. D. Jr. \& Flemming, K. D. A population-based study for 30-d hospital readmissions after acute ischemic stroke. Int. J. Neurosci. 127(4), 305-313 (2017).

24. Smith, M. A., Frytak, J. R., Liou, J. I. \& Finch, M. D. Rehospitalization and survival for stroke patients in managed care and traditional Medicare plans. Med. Care. 43(9), 902-910 (2005).

25. National Health Insurance Research Database. https://nhird.nhri.org.tw/date_cohort.html. (accessed 11 Oct 2018).

26. Liu, C. Y., Hung, Y. T. \& Chuang, Y. L. Incorporating development stratification of Taiwan townships into sampling design of large scale health interview survey. J. Health Manag. 4(1), 1-22 (2006).

27. Charlson, M. E., Pompei, P., Ales, K. L. \& MacKenzie, C. R. A new method of classifying prognostic comorbidity in longitudinal studies: Development and validation. J. Chronic Dis. 40(5), 373-383 (1987).

28. Goldstein, L. B., Samsa, G. P., Matchar, D. B. \& Horner, R. D. Charlson Index comorbidity adjustment for ischemic stroke outcome studies. Stroke 35(8), 1941-1945 (2004).

29. Sung, S. F. et al. Validity of a stroke severity index for administrative claims data research: A retrospective cohort study. $B M C$ Health Serv. Res. 16(1), 509 (2016).

30. Nakagawa, K., Ahn, H. J., Taira, D. A., Miyamura, J. \& Sentell, T. L. Ethnic comparison of 30-day potentially preventable readmissions after stroke in Hawaii. Stroke 47(10), 2611-2617 (2016).

31. Fonarow, G. C. et al. Hospital-level variation in mortality and rehospitalization for medicare beneficiaries with acute ischemic stroke. Stroke 42(1), 159-166 (2011).

32. Hsieh, C. Y., Lin, H. J., Hu, Y. H. \& Sung, S. F. Stroke severity may predict causes of readmission within one year in patients with first ischemic stroke event. J. Neurol. Sci. 372, 21-27 (2017).

33. Ko, C., Yu, T. \& Ko, T. A survey of hospital readmission in elderly patients. Hong Kong Med. J. 2, 258-262 (1996).

34. Karliner, L. S., Kim, S. E., Meltzer, D. O. \& Auerbach, A. D. Influence of language barriers on outcomes of hospital care for general medicine inpatients. J. Hosp. Med. 5(5), 276-282 (2010).

35. Feng, T. R., Hoyler, M. M., Ma, X., Rong, L. Q. \& White, R. S. Insurance status and socioeconomic markers affect readmission rates after cardiac valve surgery. J. Cardiothorac. Vasc. Anesth. 34(3), 668-678 (2020).

36. Tseng, M. C. \& Lin, H. J. Readmission after hospitalization for stroke in Taiwan: Results from a national sample. J. Neurol. Sci. 284(1-2), 52-55 (2009).

37. Weeks, W. B., Lee, R. E., Wallace, A. E., West, A. N. \& Bagian, J. P. Do older rural and urban veterans experience different rates of unplanned readmission to VA and non-VA hospitals?. J. Rural Health. 25(1), 62-69 (2009).

38. Kosar, C. M. et al. Assessment of rural-urban differences in postacute care utilization and outcomes among older US adults. JAMA Netw. Open. 3(1), e1918738 (2020).

39. Claesson, L., Gosman-Hedström, G., Lundgren-Lindquist, B., Fagerberg, B. \& Blomstrand, C. Characteristics of elderly people readmitted to the hospital during the first year after stroke. The Göteborg 70+ stroke study. Cerebrovasc. Dis. 14, 169-176 (2002).

40. Li, H. W., Yang, M. C. \& Chung, K. P. Predictors for readmission of acute ischemic stroke in Taiwan. J. Formos Med. Assoc. 110(10), 627-633 (2011).

41. White, R. S. et al. Readmission rates and diagnoses following total hip replacement in relation to insurance payer status, race and ethnicity, and income status. J. Racial Ethn. Health Disparities. 5(6), 1202-1214 (2018).

\section{Acknowledgements}

I would like to express our appreciation to Chih-Wen Chang for her data management and data analysis efforts.

\section{Author contributions}

L.J.C. participated in designing the study, data analysis, data interpretation, first drafted and revised the manuscript. H.C.L. was the PI of the study, participated in the conception and design of the study, monitored data collection, interpreted findings, drafted and revised the manuscript.

\section{Funding}

This study was funded by the Ministry of Science and Technology, Taiwan (MOST 105-2410-H-010 -012 -SS2).

\section{Competing interests}

The authors declare no competing interests.

\section{Additional information}

Supplementary Information The online version contains supplementary material available at https://doi.org/ 10.1038/s41598-022-07791-3.

Correspondence and requests for materials should be addressed to H.-C.L.

Reprints and permissions information is available at www.nature.com/reprints.

Publisher's note Springer Nature remains neutral with regard to jurisdictional claims in published maps and institutional affiliations. 
(c) (i) Open Access This article is licensed under a Creative Commons Attribution 4.0 International cc) License, which permits use, sharing, adaptation, distribution and reproduction in any medium or format, as long as you give appropriate credit to the original author(s) and the source, provide a link to the Creative Commons licence, and indicate if changes were made. The images or other third party material in this article are included in the article's Creative Commons licence, unless indicated otherwise in a credit line to the material. If material is not included in the article's Creative Commons licence and your intended use is not permitted by statutory regulation or exceeds the permitted use, you will need to obtain permission directly from the copyright holder. To view a copy of this licence, visit http://creativecommons.org/licenses/by/4.0/.

(C) The Author(s) 2022 